\title{
Desempeño laboral de los trabajadores administrativos de la municipalidad provincial de Moyobamba, San Martín Perú
}

\author{
Katherin Santillán Guzmán \\ katherin.santillan@upeu.edu.pe \\ ORCID: 0000-0002-6623-7727 \\ Escuela de administración y negocios internacionales \\ Universidad Peruana Unión \\ Renán Saavedra Sandoval \\ rsaavedrasa@unmsm.edu.pe \\ Escuela de posgrado \\ Universidad Nacional Mayor de San Marcos \\ ORCID: 0000-0002-3018-9460 \\ Tarapoto - Perú
}

\section{RESUMEN}

La presente investigación, tuvo como objetivo principal, determinar el nivel de percepción del desempeño laboral de los trabajadores de la municipalidad Provincial de Moyobamba, San Martín - Perú. El estudio realizado fue descriptivo, de diseño no experimental. Para medir la percepción del desempeño laboral, en una muestra de 60 trabajadores, se utilizó el instrument individual Work Performance (IWP), adaptado por Widyastuti \& Hidayat, que consta de las dimensiones: Rendimiento en la tarea; comportamientos contraproducentes y rendimiento en el contexto; asimismo, para obtener los resultados, se utilizó la estadística básica. Tanto el desempeño laboral, como sus dimensiones, fueron percibidas en un nivel moderado, esto implica que, los trabajadores ven como necesario una catividad planificada que permita el conocimiento previo de los ciudadanos; además el trabajador se siente con rendimiento y dedicación a su centro laboral.

Palabras clave: rendimiento en la tarea; comportamientos contraproducentes; rendimiento en el contexto. 


\title{
Labor performance of the administrative workers of the provincial municipality of Moyobamba, Lima - Peru
}

\begin{abstract}
The present investigation had as main objective, to determine the level of perception of the labor performance of the workers of the Provincial Municipality of Moyobamba, San Martín - Peru. The study carried out was descriptive, with a non-experimental design. To measure the perception of job performance, in a sample of 60 workers, the individual instrument Work Performance (IWP) was used, adapted by Widyastuti \& Hidayat, which consists of the dimensions: Performance in the task; counterproductive behaviors and performance in context; Likewise, to obtain the results, basic statistics were used. Both job performance and its dimensions were perceived at a moderate level, this implies that workers see as necessary a planned cation that allows prior knowledge of citizens; In addition, the worker feels with performance and dedication to his workplace.
\end{abstract}

Keywords: job performance; performance on task; counterproductive behaviors; performance in context.

Artículo recibido: 05 octubre. 2021 Aceptado para publicación: 02 noviembre 2021 Correspondencia: katherin.santillan@upeu.edu.pe

Conflictos de Interés: Ninguna que declarar 


\section{INTRODUCCIÓN}

En las organizaciones o instituciones, el desempeño laboral, constituye uno de los factores determinantes para el logro de las metas y objetivos trazados, por esta razón, es de gran importancia, que los trabajadores mantengan una relación entre las competencias que poseen y el cargo que desempeñan; para lo cual es necesario que se exija, el cumplimiento de requisitos que realmente permitan la selección del personal, con características que correspondan con la finalidad de la institución u organización. Específicamente, en la administración pública, es necesario que el personal que ocupa cargos, en los diferentes niveles orgánicos de la institución, posea las competencias necesarias y acordes con las funciones que deben cumplir dentro de las mismas. Por tales consideraciones, en una organización, en donde están presentes actividades de servicio a los usuarios o clientes y más aún, si esto implica administración de recursos económicos, se hace de necesidad imperativa, la realización de una evaluación del desempeño de cada trabajador, el cual permita analizar a cada servidor, en base al trabajo realizado, lo que permitirá determinar que cada trabajador, es el apropiado y corresponda con lo que realmente necesita la institución, para el logro de sus objetivos planteados.

En la misma línea, el desempeño laboral, según Chiavenato (2001), está representado por el desenvolvimiento del trabajador durante su jornada laboral, siempre y cuando lo realice con eficiencia y eficacia, es decir, que cumpla con las funciones asignadas sin ningún tipo de limitación, más que las derivadas de la empresa o institución, en cuanto a tiempo, recursos, materiales, personal, entre otros.

En términos mucho más actuales, Amaya y Conde (2015), afirman que, para ser competitivos y alcanzar el éxito, las organizaciones deben gestionar adecuadamente su talento humano, considerando los procesos a los cuales son sometidos; uno de los factores más importantes, es la evaluación del desempeño laboral.

De igual forma, en el Perú, por efecto de la globalización, las organizaciones están sujetas a cambios tecnológicos, sociales y económicos, pero también necesidades de capacitación; del proceso de adaptación a estos cambios se obtendrán las metas y logros propuestos, para ello las instituciones o empresas deben disponer de personas satisfechas con su trabajo y con la organización, ya que ello aumentará claramente su rendimiento y su calidad del servicio, haciendo de dichas organizaciones, entes más competitivos en el mercado laboral (Silva, 2017). 
Asimismo, en las municipalidades de nuestro país, encontramos un factor común, la existencia de problemas en el desempeño laboral de los trabajadores; muchos desconocen sus funciones y responsabilidades, por otra parte, están también los que sí conocen pero que no las cumplen o no las asumen con responsabilidad; ante esta situación es evidente tener como consecuencia un descenso en la efectividad y productividad laboral municipal, incrementando el malestar de la población, viéndose reflejado en los reportes de malas atenciones, escasa información a los usuarios, falta de seguimientos de trámites, etc.; todo esto es evidencia suficiente de la carencia de un lenguaje claro y único entre los trabajadores, los jefes de áreas determinadas y finalmente la población.

Con la realidad descrita líneas arriba, el presente estudio, parte del interés que se tiene de describir y visualizar los acontecimientos actuales dentro de las municipalidades, dado a que se observan deficiencias organizacionales, descoordinación, clima laboral deficiente, problemas con la remuneración, deficiencia en la calidad de servicio; para complementar el análisis objetivo sobre el problema del desempeño laboral, tenemos a bien presentar algunos de los estudios que antecedieron a la presente investigación.

Chiang y San Martín (2015), en su trabajo denominado, Análisis de la satisfacción y el desempeño laboral en los funcionarios de la municipalidad de Talcahuano Concepción Chile. Objetivo: medir la influencia del desempeño laboral sobre la satisfacción laboral de los funcionarios. Metodología: se realizó un estudio no experimental, de diseño transversal, tipo descriptivo y correlacional. La información se obtuvo de 259 funcionarios de la municipalidad a quienes se aplicó un cuestionario estandarizado, personal, anónimo y voluntario. Concluyen, que existe correlación estadísticamente significativa entre ambas variables, se destacan las correlaciones positivas entre las escalas de desempeño y satisfacción en la relación con el jefe, y las escalas de satisfacción con desempeño en la productividad.

Asimismo, Palmar y Valero (2014) en su estudio denominado, Competencias y desempeño laboral de los gerentes en los institutos autónomos dependientes de la alcaldía del municipio Mara del estado Zulia Toluca - México. Tuvo como objetivo analizar las competencias y el desempeño laboral de los gerentes en los institutos autónomos del municipio Mara. Materiales y métodos: el estudio pertenece al tipo descriptivo, de campo, transaccional y no experimental; se utilizó como instrumento un cuestionario de escala Likert, conformado por 38 ítems, con cinco alternativas de 
respuesta, aplicado a 31 sujetos que laboran en los institutos objeto de estudio. Concluyen que, al analizar las condiciones para el desarrollo de competencias, se encontró que existen condiciones necesarias para el desarrollo de las capacidades y habilidades de sus gerentes.

De igual forma, Huamán y Olarte (2017) realizaron un estudio titulado, El clima organizacional y el desempeño laboral de los trabajadores de la municipalidad distrital de Huancarani, provincia Paucartambo, región Cusco, en la gestión 2016 Cusco - Perú. Tuvo como objetivo determinar si existe relación entre clima organizacional y desempeño laboral de los trabajadores de la municipalidad distrital de Huancarani. Metodología: el estudio corresponde a la investigación cuantitativa, nivel relacional, diseño no experimental. La población de estudio estuvo conformada por los trabajadores de las diferentes áreas de la municipalidad, con 20 colaboradores. Para la recolección de datos se utilizó una encuesta de clima organizacional y formato de evaluación de desempeño laboral. Concluyen, que se obtuvo un coeficiente Rho de Spearman positivo, lo cual demuestra que existe un grado 22 de asociación lineal directa positiva y significativa entre el clima organizacional y el desempeño laboral en los trabajadores de la municipalidad distrital de Huancarani.

Por su parte, Huaraca y Ore (2016) en su investigación titulada, Desempeño laboral y la calidad de servicio del personal administrativo de la municipalidad distrital de Ascensión, Huancavelica, 2015. Huancavelica, Perú. Tuvo como objetivo determinar la relación existente entre el desempeño laboral y la calidad de servicio del personal administrativo de la municipalidad distrital. Material y métodos: se utilizó el método científico y los métodos: inductivo, deductivo, hipotético - deductivo y analítico sintético; para la recolección de datos, se utilizó la técnica de investigación documental, de fichaje y de investigación de campo; los instrumentos fueron las fichas y el cuestionario de desempeño laboral y calidad de servicio. Concluyen, que el desempeño laboral se relaciona de forma positiva y significativa con la calidad de servicio del personal administrativo de la Municipalidad Distrital de Ascensión.

Finalmente, Quispe (2015) en su estudio titulado, Clima organizacional y desempeño laboral en la municipalidad distrital de Pacucha, Andahuaylas, 2015 Apurímac, Perú. Tuvo por objetivo determinar la relación entre el clima organizacional y el desempeño laboral en la municipalidad distrital de Pacucha. Metodología: el estudio corresponde al 
enfoque cuantitativo, nivel relacional, no experimental. La población de estudio estuvo conformada por 64 trabajadores de todos los niveles de la municipalidad, para la recolección de datos se usó la técnica de la encuesta; se aplicó un cuestionario para cada variable. Concluye, que existe una relación significativa entre el clima organizacional y el desempeño laboral en la municipalidad distrital de Pacucha.

Es en este contexto, que nos planteamos como objetivo de investigación: establecer el nivel de desempeño laboral de los trabajadores administrativos de la municipalidad provincial de Moyobamba, San Martín - Perú, en sus dimensiones: rendimiento en la tarea, comportamientos contraproducentes y rendimiento en el contexto.

\section{MATERIALES Y MÉTODOS}

El tipo de investigación es descriptivo, de diseño no experimental (Sampieri, 2014) porque intentará determinar el nivel de desempeño laboral de los trabajadores administrativos de la municipalidad provincial de Moyobamba, San Martín - Perú.

La muestra, para los fines del presente trabajo de investigación, estará conformada por 60 trabajadores, que laboran en la parte administrativa de la municipalidad provincial Moyobamba, San Martín - Perú. La cantidad de trabajadores que participan en este estudio ha sido establecida por conveniencia de los investigadores.

Para medir la variable desempeño laboral, se utilizó el cuestionario Individual Work Performance (IWP), adaptado por Widyastuti \& Hidayat (2018); el cual a su vez hemos adaptado para la presente investigación. Este cuestionario consta de tres dimensiones: Rendimiento en la tarea (7 ítems), comportamientos contraproducentes (5 ítems) y rendimiento en el contexto (4 ítems). Presenta una escala de Likert: Totalmente en desacuerdo $=1$, en desacuerdo $=2$, indiferente $=3$, de acuerdo $=4$, totalmente de acuerdo $=5$

\section{RESULTADOS}

\section{Resultados 1. Percepción por preguntas.}

En cuanto a los resultados, en la tabla 1, como se puede apreciar, se identifica las percepciones de los colaboradores por preguntas de acuerdo con las dimensiones; los trabajadores refieren que se sienten con rendimiento en la tarea (2.85), cuando realizan soluciones creativas frente a problemas que tiene la institución, cuando realizan situaciones desafiantes y dedican el esfuerzo y tiempo necesarios, para los ciudadanos (3.68), observando también que el comportamiento contraproducente, con quejas de asuntos sin importancias con (3.10), y mostrando aspectos negativos con gente que no pertenece a la empresa (2.68); con un rendimiento en el trabajo, de mantener habilidades 
(2.73), para que así los ciudadanos queden satisfechos. También vemos que los comportamientos contraproducentes representan problemas que se presentaron en el trabajo con un (3.00), ya que, con el rendimiento en la tarea, ocurrieron soluciones creativas frente a problemas con un (2.58). Tanto con el rendimiento en el contexto, así como, planifiqué el trabajo de manera que pueda hacerlo en tiempo y forma con (2.60) ya que, en el rendimiento en la tarea, siguen buscando nuevos desafíos en mi trabajo.

\section{Tabla 1}

Nivel de percepción por pregunta de acuerdo con las dimensiones.

\begin{tabular}{|c|c|c|c|}
\hline Dimensiones & Ítems & Media & $\begin{array}{l}\text { Desviación } \\
\text { Estándar }\end{array}$ \\
\hline \multirow{8}{*}{$\begin{array}{l}\text { Rendimiento } \\
\text { en la tarea. }\end{array}$} & $\begin{array}{l}\text { Fui capaz de hacer bien mi trabajo porque } \\
\text { le dediqué el tiempo y el esfuerzo } \\
\text { necesario. }\end{array}$ & 2.67 & 1.35 \\
\hline & $\begin{array}{l}\text { Se me ocurrieron soluciones creativas } \\
\text { frente a los nuevos problemas. }\end{array}$ & 2.85 & 1.35 \\
\hline & $\begin{array}{l}\text { Cuando pude realicé tareas laborales } \\
\text { desafiantes. }\end{array}$ & 2.68 & 1.19 \\
\hline & $\begin{array}{l}\text { Cuando terminé con el trabajo asignado, } \\
\text { comencé nuevas tareas sin que me lo } \\
\text { pidieran. }\end{array}$ & 3.03 & 1.25 \\
\hline & $\begin{array}{l}\text { En mi trabajo, tuve en mente los resultados } \\
\text { que debí lograr }\end{array}$ & 2.80 & 1.27 \\
\hline & $\begin{array}{l}\text { Trabajé para mantener mis conocimientos } \\
\text { laborales actualizados. }\end{array}$ & 3.00 & 1.24 \\
\hline & $\begin{array}{l}\text { Siguen buscando nuevos desafíos en mi } \\
\text { trabajo. }\end{array}$ & 2.87 & 1.32 \\
\hline & Promedio & 2.84 & \\
\hline \multirow{6}{*}{$\begin{array}{l}\text { Comportamientos } \\
\text { contraproducentes. }\end{array}$} & $\begin{array}{l}\text { Me quejé de asuntos sin importancia en el } \\
\text { trabajo. }\end{array}$ & 3.10 & 1.28 \\
\hline & $\begin{array}{l}\text { Comenté aspectos negativos de mi trabajo } \\
\text { con mis compañeros. }\end{array}$ & 3.03 & 1.25 \\
\hline & $\begin{array}{l}\text { Agrandé los problemas que se presentaron } \\
\text { en el trabajo. }\end{array}$ & 3.00 & 1.30 \\
\hline & $\begin{array}{l}\text { Me concentré en los aspectos negativos del } \\
\text { trabajo, en lugar de enfocarme en las cosas } \\
\text { positivas. }\end{array}$ & 2.88 & 1.29 \\
\hline & $\begin{array}{l}\text { Comenté aspectos negativos de mi trabajo } \\
\text { con personas que no pertenecían a la } \\
\text { empresa. }\end{array}$ & 2.68 & 1.27 \\
\hline & Promedio & 2.94 & \\
\hline \multirow{5}{*}{$\begin{array}{l}\text { Rendimiento } \\
\text { en el contexto. }\end{array}$} & $\begin{array}{l}\text { Planifiqué mi trabajo de manera a que pude } \\
\text { hacerlo en tiempo y forma. }\end{array}$ & 2.60 & 1.08 \\
\hline & $\begin{array}{l}\text { Trabajé para mantener mis habilidades } \\
\text { laborales actualizadas. }\end{array}$ & 2.73 & 1.21 \\
\hline & $\begin{array}{l}\text { Participé activamente de las reuniones } \\
\text { laborales. }\end{array}$ & 3.15 & 1.18 \\
\hline & Mi panificación laboral fue óptima. & 2.97 & 1.12 \\
\hline & Promedio & 2.86 & \\
\hline
\end{tabular}




\section{Resultados 2}

\section{Percepción de desempeño laboral y sus dimensiones.}

En la tabla 2, se muestra los niveles de percepción de la variable desempeño laboral y sus dimensiones. Los resultados indican que, de los trabajadores de la municipalidad provincial de Moyobamba, San Martín - Perú, el 36\% percibe a la variable desempeño laboral en un nivel moderado, como un desempeño positivo; la primera dimensión de desempeño laboral es rendimiento en la tarea, la cual también es percibida con un nivel moderado, por la mayoría (60\%) debido a que los trabajadores presentan bajos niveles de desempeño de sus funciones; las dimensiones de comportamientos contraproducentes y rendimiento en el contexto, son percibidas en un nivel moderado (ambas 36\%), con un desempeño y rendimiento moderado.

Tabla 2

Nivel de percepción de la variable desempeño laboral y sus dimensiones

\begin{tabular}{|c|c|c|c|}
\hline & Nivel & Recuento & $\%$ \\
\hline \multirow{3}{*}{ Rendimiento en la tarea. } & Bajo & 11 & 17 \\
\hline & Moderado & 37 & 60 \\
\hline & Alto & 12 & 20 \\
\hline \multirow{3}{*}{$\begin{array}{l}\text { Comportamientos } \\
\text { contraproducentes. }\end{array}$} & Bajo & 9 & 15 \\
\hline & Moderado & 36 & 60 \\
\hline & Alto & 15 & 25 \\
\hline \multirow{3}{*}{$\begin{array}{l}\text { Rendimiento } \\
\text { contexto. }\end{array}$} & Bajo & 13 & 22 \\
\hline & Moderado & 36 & 60 \\
\hline & Alto & 11 & 18 \\
\hline \multirow{3}{*}{ Desempeño laboral } & Bajo & 10 & 17 \\
\hline & Moderado & 36 & 60 \\
\hline & Alto & 14 & 23 \\
\hline
\end{tabular}

\section{CONCLUSIONES}

La investigación identificó que, la variable desempeño laboral es considerado por la mayoría de los trabajadores como un aspecto necesario para el cumplimiento de sus funciones en la municipalidad provincial de Moyobamba, San Martín - Perú. Entre las dimensiones, la mejor valorada fue, el rendimiento en la tarea.

En la dimensión rendimiento en el contexto se percibió como moderado por el $34 \%$ de los trabajadores de la municipalidad provincial de Moyobamba, San Martín - Perú; se obtuvo un promedio de 2.73 en la escala de Likert como rendimiento en el contexto. 
Los procesos presupuestarios de una organización pueden conducir a un aumento en la participación de un trabajador con la empresa, lo que puede resultar en un mayor rendimiento en las tareas, cuando se ocurren soluciones creativas frente a problemas que tiene la institución, cuando realizan situaciones desafiantes y dediquen el esfuerzo y tiempo necesario para los ciudadanos.

Los resultados de la presente investigación, proporcionan y evidencian las características más resaltantes, de las deficiencias de los trabajadores, en su desempeño laboral; los cuales fueron observadas, mediante las dimensiones más adecuadas y precisas, constituyéndose, en un instrumento válido, para empezar a plantear las alternativas de solución más convenientes, para superar las deficiencias en el desempeño laboral de los trabajadores de una institución municipal.

La presente investigación nos permitió aprender de manera clara y precisa el valor del desempeño laboral en las instituciones públicas ya que, este aspecto es lo más importante que puede poseer una institución, el desempeño eficiente de sus trabajadores, porque no solamente constituye la garantía del debido funcionamiento institucional, sino también la imagen y el medio mismo para ofrecer servicios de calidad a los ciudadanos y/o usuarios, logrando que éstos a su vez, muestren satisfacción y respeto por la organización.

\section{REFERENCIAS BIBLIOGRAFICAS}

Alejandro, J., \& Salanova, M. (2014). Satisfacción laboral: el camino entre el crecimiento psicológico y el desempeño laboral en empresas colombianas industriales y de servicios. Universidad Psicológica, 13, 1-20. https://doi.org/10.11144/Javeriana.UPSY13-1.slcp

Alicia, O. (2010). Comportamientos extra-papel: de la colaboración a la transgresión. Revista Interamericana, 2(8), 32-53. Obtenido de: http://revista.cincel.com.co/index.php/RPO/article/view/103

Arulmani, G. (2016). Bienestar y desempeño en el trabajo: el papel del contexto. Revista Británica de Orientación y Consejería, 43(5), 611-622. https://doi.org/10.1080/03069885.2015.1069486

Bakker, A. B., \& Leiter, M. P. (2010). Compromiso laboral: un manual de teoría e investigación esenciales. Obtenido de https://psycnet.apa.org/record201006187-000 
Borman, W. C. \& Motowidlo, S. M., (1993). Ampliación del dominio del criterio para incluir elementos de desempeño contextual. Publicaciones de la Facultad de Psicología, 71-98. https://digitalcommons.usf.edu/psy_facpub/1111

Chang, M., \& San Martin, N. (2015). Análisis de satisfacción y desempeño laboral en empleados públicos del Ayuntamiento de talcahuano. Ciencia y Trabajo, 54, 159-165. Obtenido de http://dx.doi.org/10.4067/S0718-24492015000300001

Diefendorff, J., Brown, D., Kamin, A., \& Lord, R. (2002). Examinar los roles de la participación en el trabajo y la centralidad del trabajo en la predicción de los comportamientos de ciudadanía organizacional y la medición del desempeño laboral de la participación en el trabajo. Diario de comportamiento organizacional, (12), 93-108.

Freitas, M., Universidad, F., Minas, F. De y Horizonte, B. (2012). Finanzas corporativas, (2000), 677-685. https://doi.org/10.1002/9781119208372

Geraldo, L. A., Soria, J. J., Rosello, M. J., \& Buendia, K. M. (2020). Modelo factorial en la motivación laboral y desempeño docente. Caso: Institución Educativa Emblemática Isabel la Católica, Lima - Perú. Apuntes Universitarios, 11(1), 157 - 170. https://doi.org/10.17162/au.v11i1.561

Gong, Z., Chen, Y., \& Wang, Y. (2019). La influencia de la inteligencia emocional en el agotamiento y el desempeño laborales: efecto mediador del capital psicológico. Fronteras en psicología, 10

Junior, R. (2016). Desempeño laboral, satisfacción y motivación laboral: una revisión crítica de su desempeño laboral, satisfacción laboral y motivación: una revisión crítica de su relación. Revista Internacional de Avances en Gestión y Economía, (noviembre), $36-43$.

Obtenido

de https://www.researchgate.net/publication/310498763

Latham, G. P. y Locke, E. A. (2016). Una teoría del establecimiento de metas y la autorregulación del desempeño de tareas. Puerta de la investigación, 50 (abril de 1991), 212-247. https://doi.org/10.2307/258875

Lunardi, M.A., Costa, V. y Nascimento, J.C. (2019). Efectos de la participación en el trabajo, las actitudes gerenciales y el intercambio de información sobre el desempeño de los controladores en el contexto presupuestario. Revista brasileña 
de gestión empresarial Revista de gestión empresarial t, 21, 540-562. https://doi.org/10.7819/rbgn.v21i3.4000

Muda, I., Rafiki, A., \& Harahap, M. (2014). Factores que influyen en el desempeño de los empleados: un estudio sobre los bancos islámicos en la universidad de ciencias islámicas de malasia universidad de súmatela del norte. Revista Internacional de Ciencias Empresariales y Sociales, 5 (2), 73-80. Obtenido de http://ijbssnet.com/journals/Vol_5_No_2_February_2014/9.pdf

Omar, A., Vaamonde, J. D., \& Delgado, H. U. (2013). Comportamientos contraproducentes en el trabajo: diseño y validación de una escala. Diversitas, 8(2), 249. https://doi.org/10.15332/s1794-9998.2012.0002.04

Pedraza, E., Amaya, G., \& Conde, M. (2010). Desempeño laboral y estabilidad del personal administrativo contratado de la facultad de medicina de la universidad del zulia. Revista De Ver, Sociales, XVI, 493-505. Obtenido de: //www.redalyc.org/pdf/280/28016320010.pdf

Rodríguez, A., Retamal, P., Lizana, J., \& Cornejo, F. (2011). Clima y satisfacción laboral como predictores del desempeño: en una organización estatal chilena organizational climate and job satisfaction as performance. Salud \& Sociedad, 2, 219-234. Obtenido de https://www.redalyc.org/pdf/4397/439742466007.pdf Spector, P., \& Fox, S. (2010). Comportamiento laboral contraproducente y comportamiento cívico organizacional: ¿Son formas opuestas de comportamiento activo? Psicología aplicada, 59 (1), 21-39. https://doi.org/10.1111/j.1464-0597.2009.00414.x

Widyastuti, T. y Hidayat, R. (2018). Adaptación del cuestionario de desempeño laboral individual (IWPQ) en adaptación del cuestionario de desempeño laboral individual (IWPQ) en bahasa indonesia. Revista Internacional de Estudios de Investigación en Psicología, 7 (noviembre), 101-112. https://doi.org/10.5861/ijrsp.2018.3020 